\title{
The evolution of structure in $\mathrm{CH}_{5}{ }^{+}$and its deuterated analogues
}

\author{
Lindsay M. Johnson and Anne B. McCoy ${ }^{*}$ \\ Department of Chemistry, The Ohio State University, Columbus, OH 43210
}

\section{CONTENTS:}

Table S.1. Weights for each stationary point used in the calculations of the vibrationally averaged rotational constants.

Table S.2. Weights for each stationary point used in the calculations of the rotational constants.

Table S.3. Vibrationally averaged rotational Constants for all isotopologues.

\footnotetext{
*Email: mccoy@chemistry.ohio-state.edu
} 
Table S.1. Weights for each stationary point used in the calculations of the vibrationally averaged rotational constants. When a letter or combination of letters is reported, the stationary point is identical to the one indicated, by symmetry. For each stationary point of each isotopologue the weights sum to one. The $a-e$ labels refer to the binding sites, shown in Figure 1 in the text.

\begin{tabular}{|c|c|c|c|c|c|c|}
\hline $\begin{array}{c}\text { Deuterium } \\
\text { position }\end{array}$ & $C_{s}(\mathrm{I})$ & St. Dev. & $C_{2 v}$ & St. Dev. & $C_{s}(\mathrm{II})$ & St. Dev. \\
\hline $\bar{a}$ & 0.049 & 0.018 & 0.135 & 0.038 & 0.068 & 0.057 \\
\hline $\boldsymbol{b}$ & 0.007 & 0.007 & 0.008 & 0.011 & $a$ & -- \\
\hline$c$ & 0.127 & 0.047 & $a$ & -- & 0.675 & 0.111 \\
\hline$d$ & 0.817 & 0.055 & 0.857 & 0.049 & $c$ & -- \\
\hline $\boldsymbol{e}$ & $d$ & -- & $d$ & -- & 0.257 & 0.094 \\
\hline
\end{tabular}

$\mathrm{CH}_{3} \mathbf{D}_{2}^{+}$

\begin{tabular}{c|cccccc}
\hline $\begin{array}{c}\text { Deuterium } \\
\text { positions }\end{array}$ & $\boldsymbol{C}_{\boldsymbol{s}}(\mathbf{I})$ & St. Dev. & $\boldsymbol{C}_{\boldsymbol{2} \boldsymbol{v}}$ & St. Dev. & $\boldsymbol{C}_{\boldsymbol{s}}(\mathrm{II})$ & St. Dev. \\
\hline $\boldsymbol{a , \boldsymbol { b }}$ & 0.002 & 0.003 & 0.013 & 0.010 & 0.000 & 0.000 \\
$\boldsymbol{a}, \boldsymbol{c}$ & 0.002 & 0.003 & 0.003 & 0.005 & 0.008 & 0.012 \\
$\boldsymbol{a}, \boldsymbol{d}$ & 0.107 & 0.028 & 0.465 & 0.079 & 0.073 & 0.050 \\
$\boldsymbol{a}, \boldsymbol{e}$ & $a, d$ & -- & $a, d$ & -- & 0.111 & 0.060 \\
$\boldsymbol{b}, \boldsymbol{c}$ & 0.007 & 0.009 & $a, b$ & -- & $a, d$ & -- \\
$\boldsymbol{b}, \boldsymbol{d}$ & 0.055 & 0.028 & 0.026 & 0.026 & $a, c$ & -- \\
$\boldsymbol{b}, \boldsymbol{e}$ & $b, d$ & -- & $b, d$ & -- & $a, e$ & -- \\
$\boldsymbol{c}, \boldsymbol{d}$ & 0.396 & 0.036 & $a, d$ & -- & 0.098 & 0.011 \\
$\boldsymbol{c}, \boldsymbol{e}$ & $c, d$ & -- & $a, d$ & -- & 0.709 & 0.103 \\
$\boldsymbol{d , e}$ & 0.431 & 0.040 & 0.493 & 0.065 & $c, e$ & -- \\
\hline
\end{tabular}

$\mathrm{CH}_{2} \mathrm{D}_{3}^{+}$

\begin{tabular}{c|cccccc}
\hline $\begin{array}{c}\text { Hydrogen } \\
\text { positions }\end{array}$ & $\boldsymbol{C}_{\boldsymbol{s}}(\mathbf{I})$ & St. Dev. & $\boldsymbol{C}_{\boldsymbol{2} \boldsymbol{v}}$ & St. Dev. & $\boldsymbol{C}_{\boldsymbol{s}}(\mathrm{II})$ & St. Dev. \\
\hline $\boldsymbol{a}, \boldsymbol{b}$ & 0.658 & 0.025 & 0.935 & 0.119 & 0.685 & 0.067 \\
$\boldsymbol{a}, \boldsymbol{c}$ & 0.001 & 0.002 & 0.000 & 0.000 & 0.001 & 0.001 \\
$\boldsymbol{a , d}$ & 0.058 & 0.006 & 0.062 & 0.028 & 0.176 & 0.060 \\
$\boldsymbol{a}, \boldsymbol{e}$ & $a, d$ & -- & $a, d$ & -- & 0.122 & 0.056 \\
$\boldsymbol{b}, \boldsymbol{c}$ & 0.279 & 0.028 & $a, b$ & -- & $a, d$ & -- \\
$\boldsymbol{b}, \boldsymbol{d}$ & 0.001 & 0.001 & 0.004 & 0.006 & $a, c$ & -- \\
$\boldsymbol{b}, \boldsymbol{e}$ & $b, d$ & -- & $b, d$ & -- & $a, e$ & -- \\
$\boldsymbol{c}, \boldsymbol{d}$ & 0.003 & 0.002 & $a, d$ & -- & 0.012 & 0.008 \\
$\boldsymbol{c , e}$ & $c, d$ & -- & $a, d$ & -- & 0.003 & 0.006 \\
$\boldsymbol{d , e}$ & 0.000 & 0.001 & 0.000 & 0.000 & $c, e$ & -- \\
\hline
\end{tabular}

$\mathrm{CHD}_{4}^{+}$

\begin{tabular}{c|cccccc}
\hline $\begin{array}{c}\text { Hydrogen } \\
\text { position }\end{array}$ & $\boldsymbol{C}_{\boldsymbol{s}}(\mathbf{I})$ & St. Dev. & $\boldsymbol{C}_{\boldsymbol{2} \boldsymbol{v}}$ & St. Dev. & $\boldsymbol{C}_{\boldsymbol{s}}(\mathrm{II})$ & St. Dev. \\
\hline $\boldsymbol{a}$ & 0.347 & 0.079 & 0.345 & 0.063 & 0.931 & 0.105 \\
$\boldsymbol{b}$ & 0.562 & 0.102 & 0.640 & 0.077 & $a$ & -- \\
$\boldsymbol{c}$ & 0.073 & 0.021 & $a$ & -- & 0.065 & 0.037 \\
$\boldsymbol{d}$ & 0.018 & 0.021 & 0.015 & 0.013 & $c$ & -- \\
$\boldsymbol{e}$ & $d$ & -- & $d$ & -- & 0.005 & 0.005 \\
\hline
\end{tabular}


Table S.2. Weights for each stationary point used in the calculations of the rotational constants. When a letter or combination of letters is reported, the stationary point is identical to the one indicated, by symmetry and the weights in bold and italics are the stationary points that were included in the calculations of the VCI spectra. For each isotopologue the weights sum to one. The $a-e$ labels refer to the binding sites, shown in Figure 1 in the text.

$\mathrm{CH}_{3} \mathbf{D}_{2}^{+}$

\begin{tabular}{|c|c|c|c|c|c|c|}
\hline $\begin{array}{c}\text { Deuterium } \\
\text { positions }\end{array}$ & $C_{s}(\mathbf{I})$ & St. Dev. & $C_{2 v}$ & St. Dev. & $C_{s}(\mathrm{II})$ & St. Dev. \\
\hline$\overline{a, b}$ & 0.001 & 0.001 & 0.005 & 0.004 & 0.000 & 0.000 \\
\hline$a, c$ & 0.001 & 0.001 & 0.001 & 0.002 & 0.002 & 0.003 \\
\hline$a, d$ & 0.046 & 0.014 & 0.172 & 0.031 & 0.015 & 0.009 \\
\hline$a, e$ & $a, d$ & -- & $a, d$ & -- & 0.021 & 0.011 \\
\hline$b, c$ & 0.003 & 0.004 & $a, b$ & -- & $a, d$ & -- \\
\hline$b, d$ & 0.023 & 0.012 & 0.010 & 0.010 & $a, c$ & -- \\
\hline$b, e$ & $b, d$ & -- & $b, d$ & -- & $a, e$ & -- \\
\hline$c, d$ & 0.170 & 0.025 & $a, d$ & -- & 0.020 & 0.001 \\
\hline$c, e$ & $c, d$ & -- & $a, d$ & -- & 0.143 & 0.025 \\
\hline$d, e$ & 0.185 & 0.015 & 0.183 & 0.032 & $c, e$ & -- \\
\hline \multicolumn{7}{|l|}{$\mathrm{CH}_{2} \mathrm{D}_{3}^{+}$} \\
\hline $\begin{array}{c}\text { Hydrogen } \\
\text { positions }\end{array}$ & $C_{s}(\mathbf{I})$ & St. Dev. & $C_{2 v}$ & St. Dev. & $C_{s}(\mathrm{II})$ & St. Dev. \\
\hline$a, b$ & 0.259 & 0.020 & 0.353 & 0.052 & 0.157 & 0.024 \\
\hline$a, c$ & 0.000 & 0.001 & 0.000 & 0.000 & 0.000 & 0.000 \\
\hline$a, d$ & 0.023 & 0.003 & 0.023 & 0.011 & 0.039 & 0.011 \\
\hline$a, e$ & $a, d$ & -- & $a, d$ & -- & 0.030 & 0.018 \\
\hline$b, c$ & 0.110 & 0.014 & $a, b$ & -- & $a, d$ & -- \\
\hline$b, d$ & 0.000 & 0.000 & 0.001 & 0.001 & $a, c$ & -- \\
\hline$b, e$ & $b, d$ & -- & $b, d$ & -- & $a, e$ & -- \\
\hline$c, d$ & 0.001 & 0.001 & $a, d$ & -- & 0.003 & 0.003 \\
\hline$c, e$ & $c, d$ & -- & $a, d$ & -- & 0.001 & 0.002 \\
\hline$d, e$ & 0.000 & 0.000 & 0.000 & 0.000 & $c, e$ & -- \\
\hline
\end{tabular}


Table S.3. Vibrationally averaged rotational Constants for all isotopologues. Separate rotational constants were evaluated for each of the three stationary points, depicted in Figure 1 of the text. These were obtained by evaluating the weighted average of the vibrationally averaged rotational constants for each of the isotopomers of that isotopologue. The weights that were used for these calculations are reported in Table S.1 and the Hamiltonian for which the rotational constants are derived is given in Eq. (2) of the text.

\begin{tabular}{|c|c|c|c|c|c|c|}
\hline $\mathrm{CH}_{5}^{+}$ & $C_{s}(\mathrm{I})$ & St. Dev. & $C_{2 v}$ & St. Dev. & $C_{s}(\mathrm{II})$ & St. Dev. \\
\hline$B_{a a}$ & 3.892 & 0.010 & 3.895 & 0.010 & 3.890 & 0.010 \\
\hline$B_{a b}$ & 0.000 & & 0.000 & & 0.000 & \\
\hline$B_{a c}$ & 0.000 & & 0.000 & & -0.012 & 0.001 \\
\hline$B_{b b}$ & 3.861 & 0.010 & 3.867 & 0.010 & 3.864 & 0.010 \\
\hline$B_{b c}$ & 0.012 & 0.001 & 0.000 & & 0.000 & \\
\hline$B_{c c}$ & 3.850 & 0.010 & 3.841 & 0.010 & 3.849 & 0.010 \\
\hline $\mathrm{CH}_{4} \mathrm{D}^{+}$ & $C_{s}(\mathrm{I})$ & St. Dev. & $C_{2 v}$ & St. Dev. & $C_{s}(\mathrm{II})$ & St. Dev. \\
\hline$B_{a a}$ & 3.707 & 0.020 & 3.726 & 0.031 & 3.713 & 0.024 \\
\hline$B_{a b}$ & 0.160 & 0.055 & 0.137 & 0.018 & 0.134 & 0.047 \\
\hline$B_{a c}$ & 0.044 & 0.021 & 0.000 & & 0.031 & 0.018 \\
\hline$B_{b b}$ & 3.095 & 0.016 & 3.095 & 0.010 & 3.085 & 0.021 \\
\hline$B_{b c}$ & 0.019 & 0.009 & 0.000 & & 0.010 & 0.006 \\
\hline$B_{c c}$ & 3.070 & 0.014 & 3.051 & 0.021 & 3.074 & 0.017 \\
\hline $\mathrm{CH}_{3} \mathrm{D}_{2}{ }^{+}$ & $C_{s}(\mathrm{I})$ & St. Dev. & $C_{2 v}$ & St. Dev. & $C_{s}(\mathrm{II})$ & St. Dev. \\
\hline$B_{a a}$ & 3.157 & 0.031 & 3.154 & 0.044 & 3.151 & 0.033 \\
\hline$B_{a b}$ & 0.029 & 0.032 & 0.001 & 0.011 & 0.044 & 0.032 \\
\hline$B_{a c}$ & 0.021 & 0.051 & 0.051 & 0.054 & 0.035 & 0.048 \\
\hline$B_{b b}$ & 2.791 & 0.023 & 2.811 & 0.031 & 2.795 & 0.025 \\
\hline$B_{b c}$ & 0.072 & 0.023 & 0.008 & 0.008 & 0.067 & 0.033 \\
\hline$B_{c c}$ & 2.547 & 0.026 & 2.529 & 0.030 & 2.548 & 0.016 \\
\hline $\mathrm{CH}_{2} \mathrm{D}_{3}{ }^{+}$ & $C_{s}(\mathrm{I})$ & St. Dev. & $\overline{C_{2 v}}$ & St. Dev. & $C_{s}(\mathrm{II})$ & St. Dev. \\
\hline$B_{a a}$ & 2.555 & 0.013 & 2.551 & 0.029 & 2.555 & 0.006 \\
\hline$B_{a b}$ & 0.001 & 0.006 & 0.003 & 0.012 & 0.002 & 0.014 \\
\hline$B_{a c}$ & 0.005 & 0.020 & 0.008 & 0.031 & 0.025 & 0.006 \\
\hline$B_{b b}$ & 2.552 & 0.003 & 2.545 & 0.001 & 2.547 & 0.029 \\
\hline$B_{b c}$ & 0.032 & 0.008 & 0.054 & 0.003 & 0.019 & 0.029 \\
\hline$B_{c c}$ & 2.267 & 0.015 & 2.278 & 0.030 & 2.272 & 0.034 \\
\hline $\mathrm{CHD}_{4}{ }^{+}$ & $C_{s}(\mathrm{I})$ & St. Dev. & $\overline{C_{2 v}}$ & St. Dev. & $C_{s}(\mathrm{II})$ & St. Dev. \\
\hline$B_{a a}$ & 2.191 & 0.008 & 2.192 & 0.007 & 2.185 & 0.003 \\
\hline$B_{a b}$ & 0.000 & 0.002 & 0.000 & & 0.008 & 0.002 \\
\hline$B_{a c}$ & 0.000 & 0.001 & 0.000 & & 0.003 & 0.001 \\
\hline$B_{b b}$ & 2.175 & 0.008 & 2.175 & 0.007 & 2.181 & 0.008 \\
\hline$B_{b c}$ & 0.007 & 0.006 & 0.005 & 0.007 & 0.010 & 0.002 \\
\hline$B_{c c}$ & 2.173 & 0.006 & 2.171 & 0.003 & 2.173 & 0.005 \\
\hline $\mathrm{CD}_{5}{ }^{+}$ & $C_{s}(\mathrm{I})$ & St. Dev. & $C_{2 v}$ & St. Dev. & $C_{s}(\mathrm{II})$ & St. Dev. \\
\hline$B_{a a}$ & 1.980 & 0.006 & 1.981 & 0.006 & 1.979 & 0.006 \\
\hline$B_{a b}$ & 0.000 & & 0.009 & & 0.000 & \\
\hline$B_{a c}$ & 0.000 & & 0.000 & & -0.006 & 0.001 \\
\hline$B_{b b}$ & 1.964 & 0.006 & 1.966 & 0.006 & 1.965 & 0.006 \\
\hline$B_{b c}$ & 0.006 & 0.001 & 0.000 & & 0.000 & \\
\hline$B_{c c}$ & 1.956 & 0.006 & 1.953 & 0.006 & 1.956 & 0.006 \\
\hline
\end{tabular}

